\title{
Moving Particle Semi-implicit Method in Simulating Water-Oil Penetration
}

\author{
Y. Yulianto, A. P. A. Mustari*, M. Ilham, A. N. Hidayati, S. Hatmanti \\ Nuclear Physics and Biophysics Research Division, Physics Department, \\ Faculty of Mathematics and Natural Science, Bandung Institute of Technology \\ Jl. Ganesha 10, Bandung 40132, Indonesia
}

( Received: August 23, 2018, Revised: November 28, 2018, Accepted: August 23, 2019 )

\begin{abstract}
In this study, the simulation of water-oil penetration has been performed by using the MPS method. The MPS method has been utilized to simulate the two-dimensional water-oil penetration and to observe the interaction of those liquids. This water-oil penetration phenomenon can be applied to the case of nuclear reactors. This study was performed to observe the liquid flow motion and its interaction between two different immiscible liquids with experiment and simulation. The obtained results of MPS method show an acceptable agreement with the experiment results. Those obtained results explain that the MPS method used in this study has a good enough capability to simulate the water-oil penetration phenomenon, which can be implemented in analyzing the melted reactor core.
\end{abstract}

Keywords: immiscible liquid, meshless, MPS method, water-oil penetration

\section{INTRODUCTION}

The usage of nuclear technology in providing energy has significant development. On the other hand, this development faces many problems to be solved. Several accidents in nuclear power plant, such as Chernobyl, Three Miles Island, and Fukushima, make us consider seriously about the usage of nuclear technology, especially reactor safety. It is needed a better understanding of how the accidents occur. The Fukushima boiling water reactor is one of the reactor accidents which can be an essential phenomenon in picturing how the movement of the molten fuel liquid in the core during accident. The melting process of fuel and control rods causes system to fail in the reactor core. This melting is predicted to be accumulated in the lower plenum.

Based on those facts, an investigation is needed to explain the behavior of melted reactor core materials when reactor core accidents occur. Conducting experiment is sometimes costly thus simulation is much more favorable for some

\footnotetext{
*Corresponding author.

E-mail address: pramutadi@fi.itb.ac.id
}

conditions. In different circumstances, the conventional simulation methods, which are dependent of empirical correlations, have difficulty in analyzing several melted core phenomena, such as stratification, free-surface flow, and phase transition due to dynamic change of the surface. Therefore, a new method is needed to solve those problems, which does not depend on empirical correlations and can be implied to analyze the severe accidents of reactor core. In order to reduce the cost of experiments, computational simulation can be an alternative way.

Moving Particle Semi-implicit (MPS) method, a meshless particle method which was first introduced by Koshizuka and Oka [1], is one of the methods that can be utilized to simulate the melted core phenomena. The MPS method can be used to analyze incompressible free surface flow. This method has been applied successfully in nuclear engineering to explain some liquid phenomena $[2,3,4]$.

This study attempts to combine the study of MPS method conducted by Ilham et al. [5] and by Yulianto et al. [6], where Ilham et al. explained the relocation of non-compressed liquid and Yulianto et 
al. explained about stratification behavior of immiscible liquid. In this present study, it is utilized the two-dimensional MPS method to simulate the process of liquid which penetrates into the other liquid and to observe its interaction process. It was used the immiscible liquid in order to avoid the liquids to be mixed. The simulation is also validated with the simple experiments.

\section{THEORY}

\section{Governing Equation}

For incompressible flow, the commonly used governing equation is Navier-Stokes equation, which can be described as [1,7]

$$
\begin{aligned}
& \frac{D r}{D t}=0 \\
& \frac{D \stackrel{1}{D}}{D t}=-\frac{1}{r} \tilde{\mathrm{N}} P+n \tilde{\mathrm{N}}^{2} \stackrel{\mathrm{r}}{u}+\stackrel{\mathrm{r}}{g}
\end{aligned}
$$

where $\rho$ is the density, $t$ is the time, $\stackrel{t}{u}$ is velocity vector, $\tilde{\mathrm{N}}$ is the gradient, $P$ is the pressure, $n$ is the

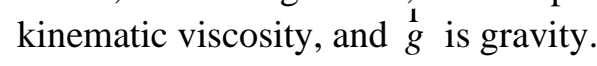

\section{Moving Particle Semi-Implicit method}

Moving Particle Semi-implicit method is a meshless particle method in analyzing incompressible flow, where the particle interaction is defined according to its position related to the reference particle. From the interaction force between two nearest particles, the particle motion can be approximated by using weight function related to the distance.

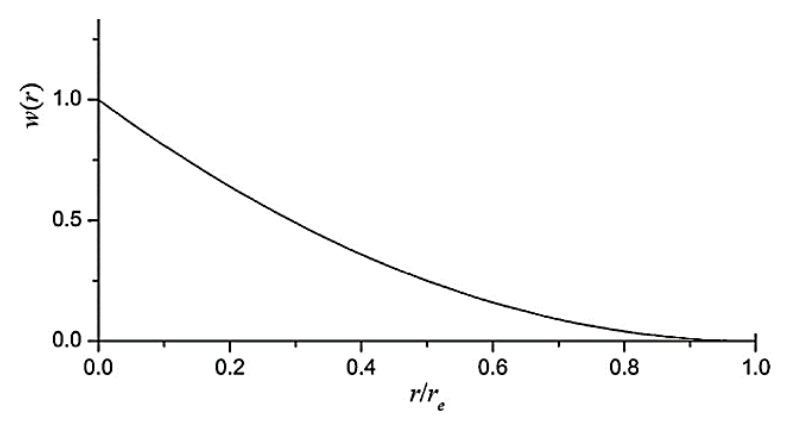

Figure 1. Weight function [7].

The weight function used commonly in the MPS method is

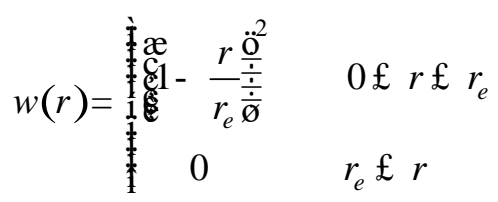

Here, $r$ is the distance between two particles and $r_{e}$ is the cut-off radius for limited interaction $[1,7]$. When the distance between two particles is higher than the cut-off radius, the weight function approaches to be zero [1], as shown in Fig. 1. The particle number density, which equals to the liquid density, on $i$-particle position can be approximated by using

$n_{i}=\underset{j^{1} i}{\stackrel{\circ}{a}} w\left(\left|\begin{array}{ll}\mathrm{r} & r_{j} \\ r_{i}\end{array}\right|\right)$

where $\stackrel{1}{r_{i}}$ and ${ }^{1} r_{j}$ are position vectors of $i$ and $j$ particles [1,7].

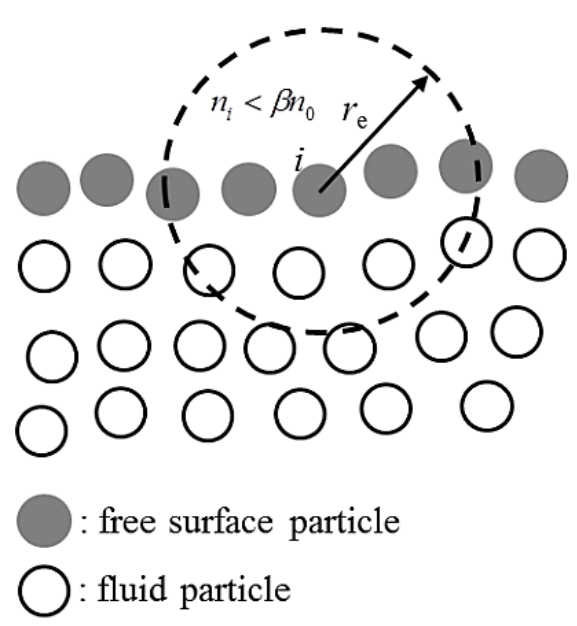

Figure 2. Free surface boundary condition [7].

The gradient, divergence, and Laplacian models can be calculated by using:

$$
\begin{aligned}
& \langle\stackrel{\mathrm{r}}{\mathrm{N}} f\rangle_{i}=\frac{d}{n^{0}} \underset{j^{1}}{\mathrm{a}} \frac{f_{j}-\hat{f_{i}}}{i\left|r_{j}-r_{i}\right|^{\mathrm{r}}}\left(r_{j}^{\mathrm{r}}-\stackrel{\mathrm{r}}{r_{i}}\right) w\left(\left|r_{j}-r_{i}^{\mathrm{r}}\right|\right) \\
& \left\langle\stackrel{\mathrm{r}}{\mathrm{N}} x_{j}^{\mathrm{r}}\right\rangle_{i}=\frac{d}{n^{0}} \underset{j^{\prime}}{\mathrm{a}} \frac{j_{j}-j_{i}}{\left|r_{j}^{\mathrm{r}}-r_{i}\right|^{2}}\left(r_{j}-\stackrel{\mathrm{r}}{r_{i}}\right) w\left(\left|r_{j}-\stackrel{r}{r}_{i}\right|\right) \\
& \left\langle\tilde{\mathrm{N}}^{2} f\right\rangle_{i}=\frac{2 d}{l n^{0}}{\stackrel{\mathrm{a}}{j^{1}}(i}_{j}\left(f_{j}-f_{i}\right) w\left(\left|\begin{array}{ll}
\mathrm{r} & r_{j}-r_{i}
\end{array}\right|\right)
\end{aligned}
$$

where $d$ is the number of spatial dimensions, $n^{0}$ is the initial particle number density, $f_{j}$ is the scalar of the $j$-particle at $r_{j}, \hat{f}_{i}$ is the minimum value of the scalar quantity in the effective radius of the $i$ target particle, and $\lambda$ is the chosen parameter to make the obtained Laplacian model to be proportional to the analytical solution [8]. The value of $\lambda$ can be calculated by using

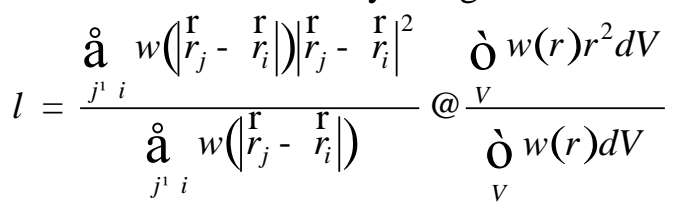

To preserve the incompressible conditions for internal particles, the particle number density is set to be constant, while it decreases for the particles located on the free surface. The observed particles, 
regarded as free surface particles shown in Fig. 2, should meet the condition of

$$
n_{i}<b n^{0}
$$

The parameter $\beta$ is the constant value, which satisfies $b<1$ [1,7]. The viscosity term was first implicitly calculated by using the discretized Laplacian model, which can be described as $[7,8]$

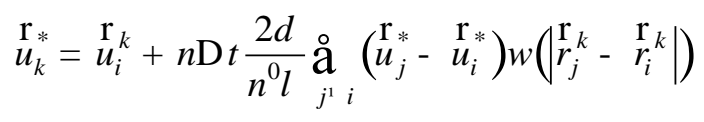

where the superscripts * and $k$ represent the temporary values and the values at the last time step respectively. The new temporal velocity and its corresponding position can be calculated by $[7,8]$

$$
\begin{aligned}
& \stackrel{\mathrm{r}}{u * *}_{*}=\stackrel{\mathrm{r}}{u}^{*}+\mathrm{D} t \stackrel{\mathrm{r}_{g}}{\mathrm{r}_{* *}} \\
& r^{\mathrm{r}}=r^{k}+\mathrm{D} t \stackrel{r}{u}^{* *}
\end{aligned}
$$

After the Poisson equations of pressure are solved, the pressure term on the eq. (2) is calculated. The velocity and position are corrected by using $[7,8]$

$$
\begin{aligned}
& \stackrel{\mathrm{r}}{k+1}^{k+}=\stackrel{\mathrm{r}}{* * *}^{*}+\mathrm{D} t\left(-\frac{1}{r^{0}} \tilde{\mathrm{N}} P\right) \\
& r^{r_{k+1}}=r^{\mathrm{r}_{* *}}+(\mathrm{D} t)^{2}\left(-\frac{1}{r^{0}} \tilde{\mathrm{N}} P\right)
\end{aligned}
$$

The flowchart of the source code used in this study can be seen in Fig. 3. The calculations were performed numerically, i.e. explicitly by using the finite difference method and implicitly by using the Crank-Nicholson method.

\section{SIMULATION AND EXPERIMENT}

Simulation. The initial condition for twodimensional simulation is shown in Fig. 4. In this study, the used particles are 4365 . The density and the kinematic viscosity are treated as the average values of each used liquid in the liquid interface, as used in references [7,8]. The liquid parameters [6] can be seen in Table 1 .

Table 1. Liquid parameters [6].

\begin{tabular}{ccc}
\hline \multicolumn{1}{c}{ Case } & \multicolumn{2}{c}{$\begin{array}{c}\text { Parameter } \\
\text { kinematic viscosity } \\
\left(\mathrm{kg} / \mathrm{mm}^{3}\right)\end{array}$} \\
\hline $\begin{array}{c}\text { Case } 1 \\
\quad \text { Liquid A }\end{array}$ & \\
$\quad \begin{array}{l}\text { (Cooking oil) } \\
\text { Liquid B } \\
\text { (Freshwater) }\end{array}$ & 890.13 & 53.146 \\
$\begin{array}{l}\text { Case 2 } \\
\quad \text { Liquid A } \\
\text { (Lubricant oil) }\end{array}$ & 848.30 & 1.004 \\
$\quad \begin{array}{l}\text { Liquid B } \\
\text { (Freshwater) }\end{array}$ & 1000 & 143.70 \\
$\begin{array}{l}\text { Case 3 } \\
\quad \text { Liquid A } \\
\text { (Lubricant oil) }\end{array}$ & 848.30 & 1.004 \\
$\quad \begin{array}{l}\text { Liquid B (Cooking } \\
\text { oil) }\end{array}$ & 890.13 & 143.70 \\
\hline
\end{tabular}

Experiment. In this study, it is utilized three types of immiscible liquids, i.e. freshwater, cooking oil, and lubricant oil. Those three types of liquid are paired with a two-liquid combination. It was set that the high-density immiscible liquid (liquid B) falls down smoothly into the low-density immiscible liquid (liquid A). First, liquid A was placed in the box container with $76 \mathrm{~mm} \times 20 \mathrm{~mm} \times 50 \mathrm{~mm}$ of dimensions. The height of liquid $\mathrm{A}$ was set $20 \mathrm{~mm}$ from the bottom of the container. Next, liquid B was fallen down to the container through the wall of container smoothly. It is kept the liquid B does not make some splashes in entering the liquid A. It was continued until all combinations in Table 1 were performed. 


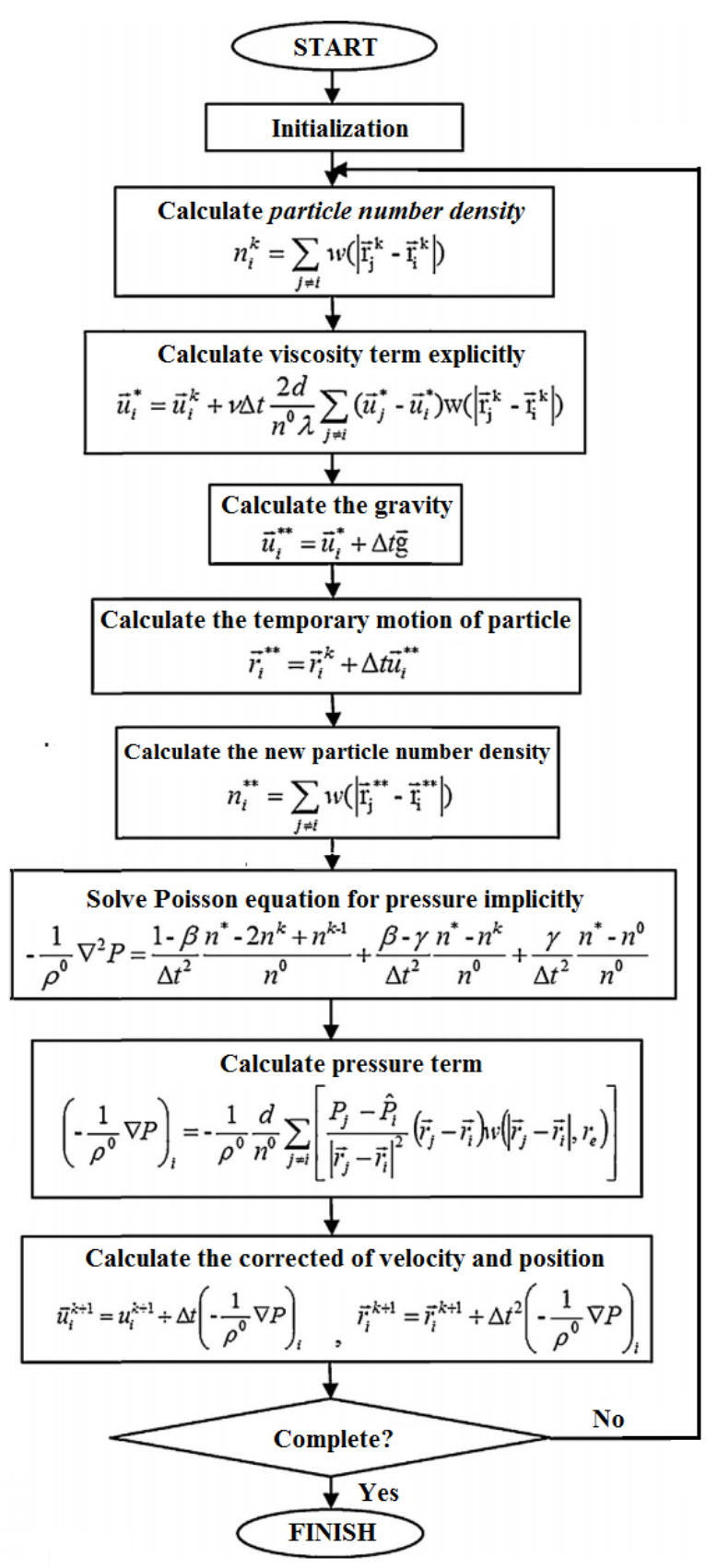

Fig. 3. Flowchart of MPS method [8].

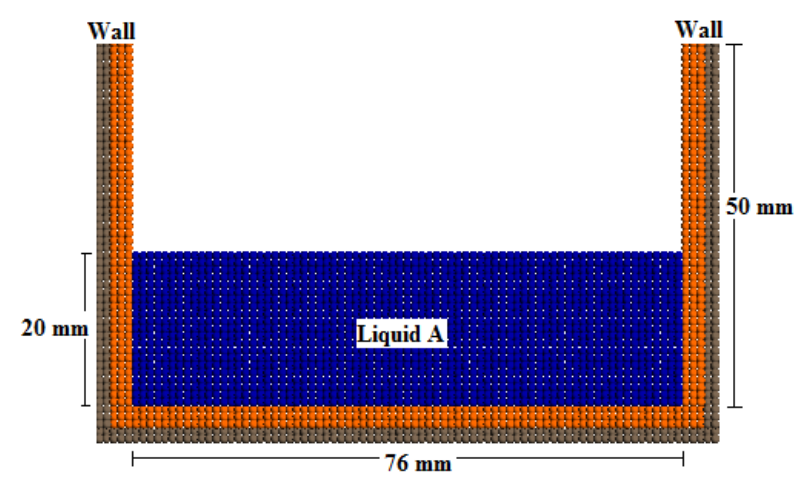

(a) simulation

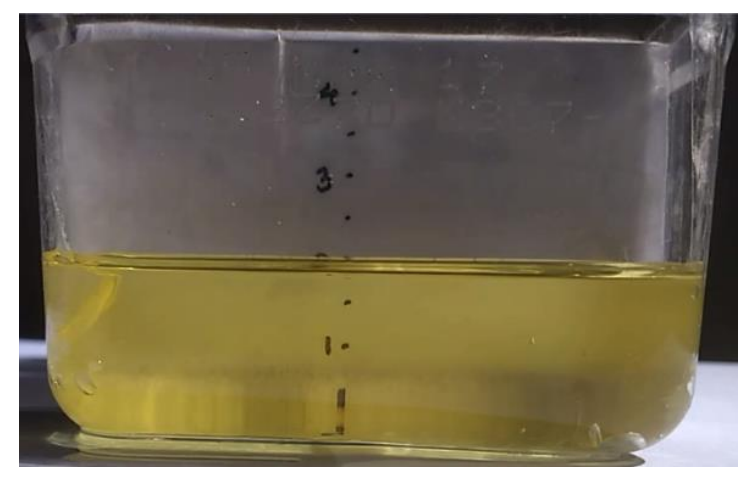

(b) experiment

Figure 4. Set up of particles in initial condition of this study. 


\section{RESULTS AND DISCUSSION}

In this study, the simulations were carried out first. Next, the experiments were performed to validate the simulation. The obtained results for both simulation and experiment are displayed in Fig. 5-7. In Fig. 5, it can be seen the results of experiment and simulation for the cooking oil and freshwater. The results of experiment and simulation for the lubricant oil and freshwater can be seen in Fig. 6 . Fig. 7 shows the results of experiment and simulation for the cooking oil and the lubricant oil.

From Fig. 5, it can be seen that the freshwater falls down smoothly into the cooking oil. The high-density liquid moves down under the lowdensity liquid. When striking the corner of the container, the freshwater undergoes turbulence phenomenon that makes the shape of freshwater inside the cooking oil become just like a big bubble. In the simulation, the shape of the freshwater inside the cooking oil is not like a big bubble. It can be also seen that, in the experiment, the freshwater moves only in the bottom of the container with bubble shape, but in simulation, the freshwater can reach the surface of cooking oil. In addition, when observing how long time the liquid penetrates inside the other liquid, it is obtained that the simulation moves faster than the experiment. Those results in Fig. 5 are similar to the results in Fig. 6, which explain the case of the lubricant oil and freshwater.

From those results, it can be indicated that in this case, after 0.5 second, simulation and experiment are roughly in a good agreement. It is predicted that the differences between the experiment and the simulation are influenced by the turbulence phenomenon occurred inside the liquid. The current source code used in this study has not been improved to tackle such a case. It can be indicated that it is most likely to be able to approach the experiment when the calculation of turbulence is implied in the current source code. It is needed the further investigation to explain those simulation results.
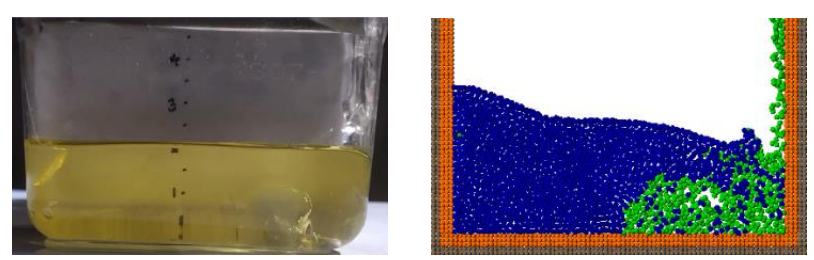

a) $t=0.27$ second

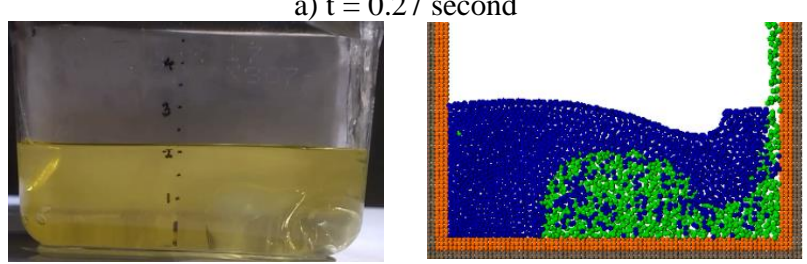

b) $\mathrm{t}=0.40$ second

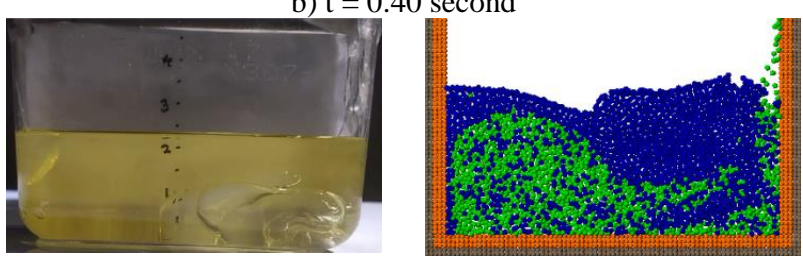

c) $\mathrm{t}=0.69$ second

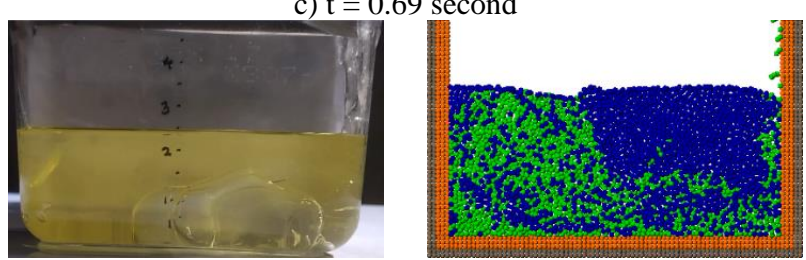

d) $\mathrm{t}=0.93$ second

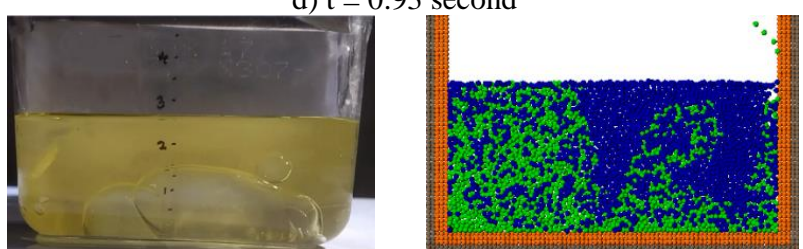

e) $\mathrm{t}=1.26$ second 


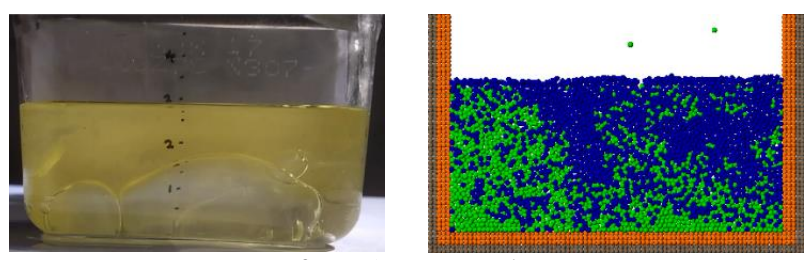

f) $\mathrm{t}=1.76$ second

Figure 5. The results of experiment (left-hand side) and simulation (right-hand side) for the cooking oil and freshwater.

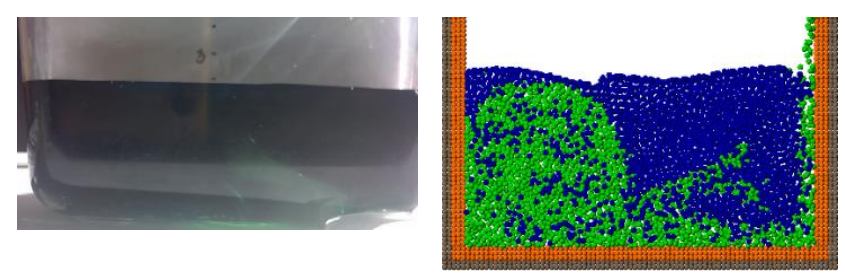

a) $t=0.63$ second

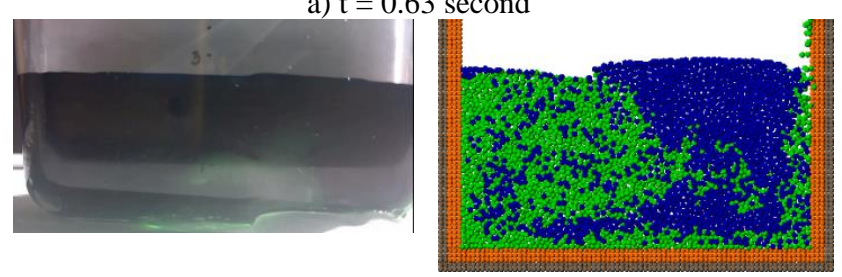

b) $t=0.92$ second

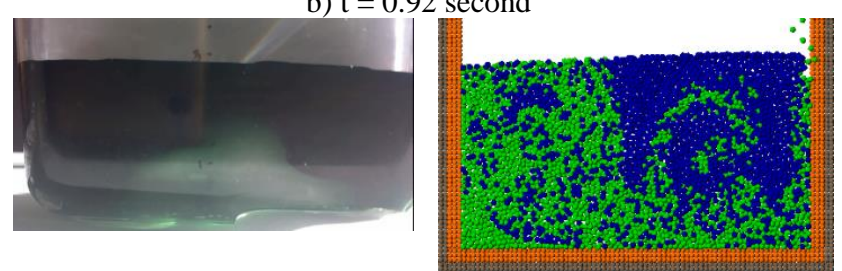

c) $\mathrm{t}=1.26$ second

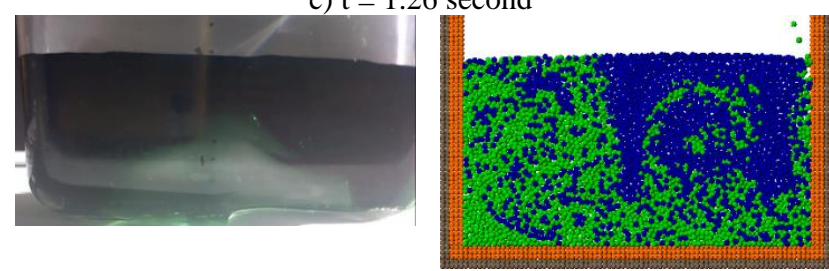

d) $\mathrm{t}=1.32$ second

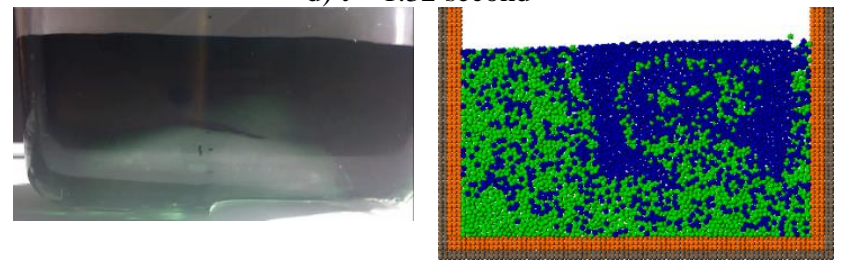

e) $\mathrm{t}=1.53$ second

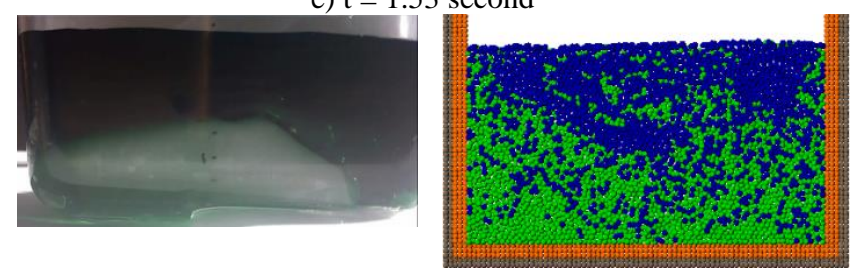

f) $\mathrm{t}=1.86$ second

Figure 6. The results of experiment (left-hand side) and simulation (right-hand side) for the lubricant oil and freshwater.

For the cooking oil and the lubricant oil, the simulation results have been roughly achieved an acceptable agreement with the experiment results, as shown in Fig. 7. The experiment shows that the cooking oil moves at the bottom of the container inside the lubricant oil without reaching the surface of the lubricant oil. However, by the simulation, it is shown that the cooking oil reaches the surface of the lubricant oil. In addition, inside the lubricant oil, the cooking oil is also very difficult to reach the other wall of the container by moving at the bottom of the container. It can be indicated that the turbulence has a very strong effect to make the spreading process move slow. It is needed to imply the calculation of 
turbulence in the current source code to obtain a better result in approaching the experiment. The
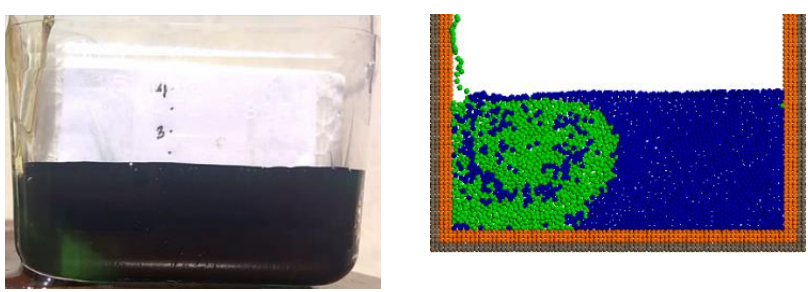

a) $\mathrm{t}=0.66$ second
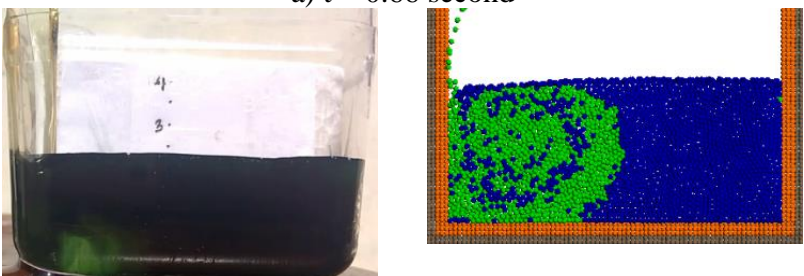

b) $\mathrm{t}=0.83$ second
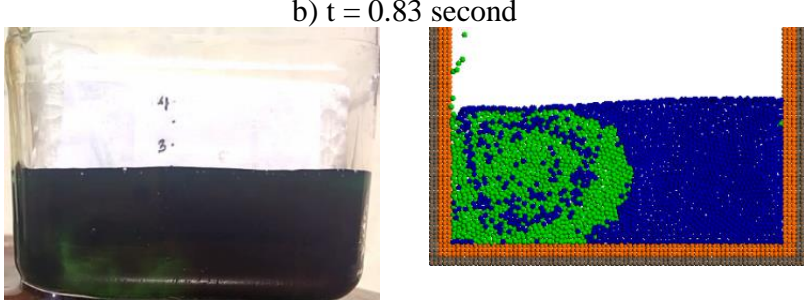

c) $\mathrm{t}=0.92$ second
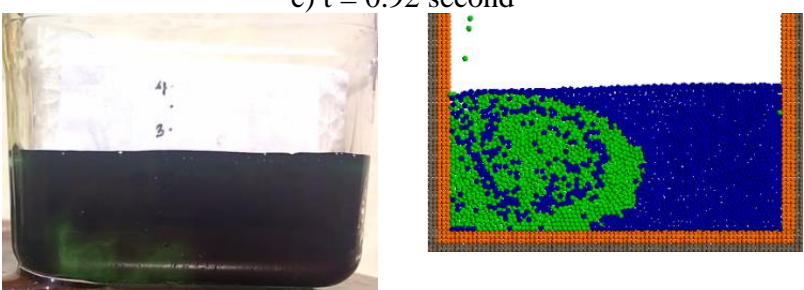

d) $\mathrm{t}=1.16$ second
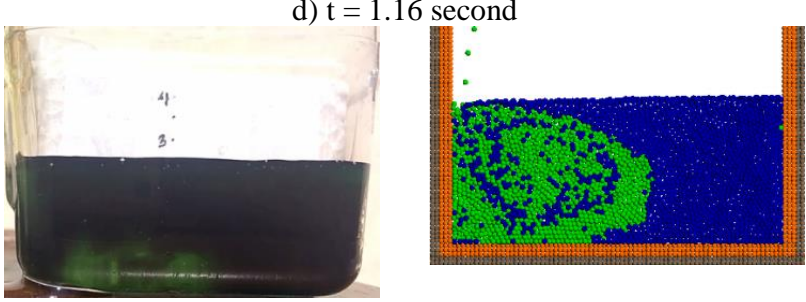

e) $\mathrm{t}=1.29$ second
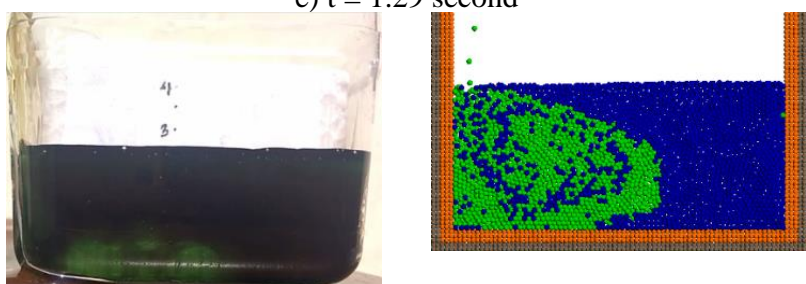

e) $t=1.46$ second

Figure 7. The results of experiment (left-hand side) and simulation (right-hand side) for the cooking oil and the lubricant oil. 


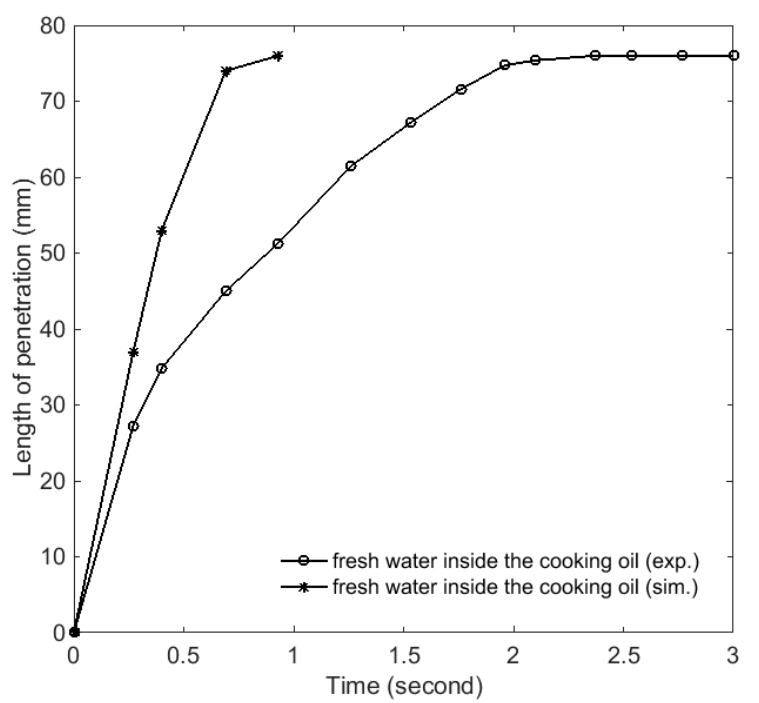

Figure 8 . The length of penetration of freshwater inside the cooking oil.

Finally, to understand how fast the liquid penetrates inside the other liquid obtained by experiment and simulation, it is also shown the length of penetration of the liquid inside the other liquid as shown in Fig. 8-10. Fig. 8 shows the length of penetration of freshwater inside the cooking oil. The length of penetration of freshwater inside the lubricant oil can be seen in Fig. 9. Fig. 10 shows the length of penetration of cooking oil inside the lubricant oil. From Fig. 8, it can be seen that the movement of simulation is faster than that of the experiment. The similar differences are also found when heading to Fig. 9 and Fig. 10. It explains implicitly that the turbulence calculation is very important in liquid penetration.

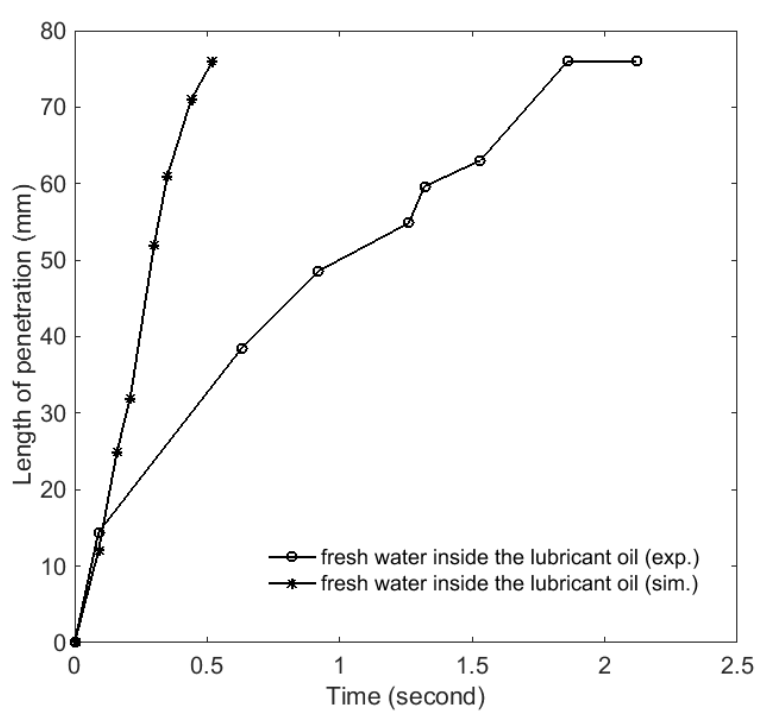

Figure 9. The length of penetration of freshwater inside the lubricant oil.

Based on those obtained results above, the simulation results of this study have achieved an acceptable agreement with the experiment results. On the other hand, it can be indicated that the turbulence phenomenon has a very strong effect on liquid penetration. It is predicted that the turbulence phenomenon affects the calculation in the pressure term so that it affects the velocity of the liquid when penetrating inside the other liquid. The turbulence phenomenon is needed to be implied in the source code in order to improve the capability of the source code in solving liquid penetration phenomena. In addition, some appropriate parameters should be added so that the turbulence phenomena in the liquid can be explained. The simulation is most likely to be able to approach the experiment if the improvement of the source code is performed. 


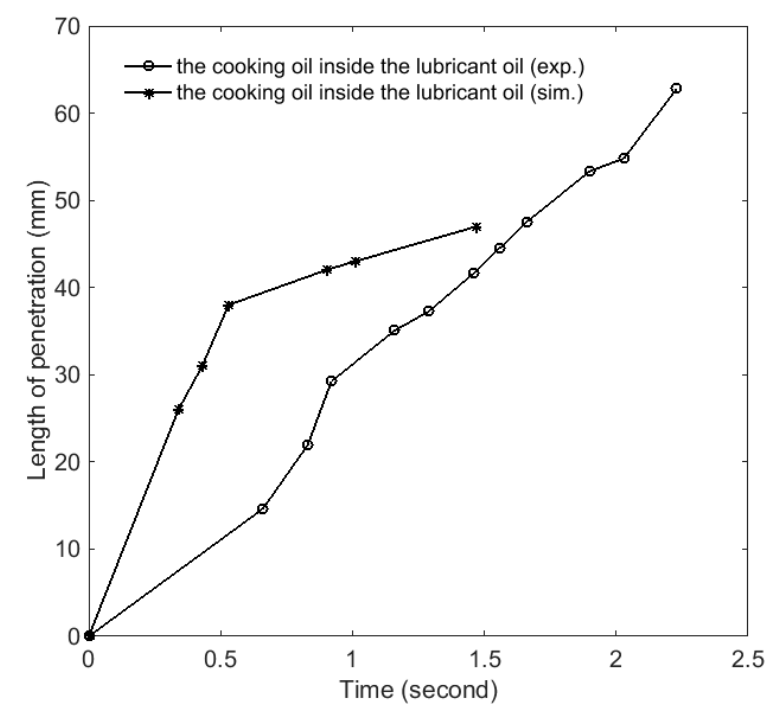

Figure 10. The length of penetration of cooking oil inside the lubricant oil.

\section{CONCLUSION}

In this study, it has been performed some simulations of water-oil penetration. The MPS method has been utilized to accommodate those simulations. The results of the simulation have been validated with the results of the simple experiment. The obtained results show that the simulation results achieve an acceptable agreement with the experiment results. The two-dimensional simulation results indicate that the MPS method can be used to analyze the behavior of the liquid to penetrate inside the other liquid with different density and/or different kinematic viscosity. This model can later be used to explain large-scale simulations such as melted reactor core.

\section{ACKNOWLEDGMENT}

This study is fully funded by the Ministry of Research, Technology, and Higher Education 2018, Indonesia.

\section{REFERENCES}

[1] S. Koshizuka and Y. Oka, Moving particle semi-implicit method for fragmentation of incompressible fluid, Nuclear Science and Engineering 123, 421, 1996.
[2] S. Koshizuka and Y. Oka, Application of moving particle semi-implicit method to nuclear reactor safety, Comput. Fluid Dyn. J. 9, 366, 2001.

[3] T. Kawahara and Y. Oka, Ex-vessel molten core solidification behavior by moving particle semi-implicit method, J. Nucl. Sci. Technol. 49, 1156, 2012.

[4] A. P. A. Mustari et al., 3D simulation of eutectic interaction of $\mathrm{Pb}-\mathrm{Sn}$ system using Moving Particle Semi-implicit (MPS) method, Annals of Nuclear Energy 81, 26, 2015.

[5] M. Ilham, Y. Yulianto, and A. P. A. Mustari, Simulation on relocation of non-compressed fluid flow using Moving Particle SemiImplicit (MPS) method, IOP Conf. Ser.: Mater. Sci. Eng. 407, 012100, 2018.

[6] Y. Yulianto et al., Moving Particle Semiimplicit (MPS) utilization in analyzing the stratification behavior of immiscible liquid, IOP Conf. Ser.: Mater. Sci. Eng. 407, 012189, 2018.

[7] G. Li et al., Study on melt behavior in a BWR lower head by MPS method and MELCOR code, Doctoral Thesis, Waseda University Graduate School of Advanced Science and Engineering, 2015.

[8] G. Li et al., Experiments and MPS analysis of stratification behavior of two immiscible fluids, Nucl. Eng. Des. 265, 210, 2013. 